\title{
3C seismic data processing and interpretation: a case study from Carpathian Foredeep basin
}

\author{
Żaneta Szymańska-Małysa ${ }^{1}$ (D) $\cdot$ Paweł Dubiel $^{1}$
}

Received: 10 May 2019 / Revised: 16 September 2019 / Accepted: 30 September 2019 / Published online: 14 October 2019

(c) The Author(s) 2019

\begin{abstract}
Chałupki Dębniańskie seismic profile 2D-3C is located in Carpathian Foredeep basin, Poland, and is an object of interest for geologists and geophysicists due to the presence of gas-bearing layers. Multicomponent seismic plays a significant role in supporting reservoir analysis related to accumulations of crude oil and natural gas. The purpose of the research was the optimal processing workflow design, which integrated seismic images of three-component 2D seismic line (2D-3C seismic). A complete processing flow for vertical and both horizontal components was conducted to obtain stacks and prestack gathers with preserved amplitude relations (RAP processing). The main issue of the research was the interpretation of S-wave velocity, which was not provided by well log data. The obtained results increased the reliability of seismic interpretation within Chałupki Dębniańskie area. The research provided valuable information regarding amplitude anomalies and helped in the verification of the potential gas accumulations. Several reservoir analysis tools were tested, including seismic attributes and AVO analysis. Conducted research confirmed the existence of reservoir which is characterized by good reservoir parameters.
\end{abstract}

Keywords Seismic processing $\cdot$ Multicomponent seismic $\cdot$ DHI $\cdot$ AVO

\section{Introduction}

PP-wave surveys are commonly used in geophysics, both in Poland and in the world, in contrast to full-wave recordings, which processing and interpretation are more demanding. Due to increasing difficulties in the prospection of oil or natural gas reservoirs, the industry began to look for methods that could help in the exploration of deposits where conventional P-wave imaging was insufficient (Farfour and Yoon 2016).

To determine the relevance of converted wave recording, we should refer to the basic characteristics of S-wave propagation in the rock medium. These include:

- much lower S-wave velocity than P-wave velocity;

Żaneta Szymańska-Małysa

zmalysa@agh.edu.pl

Paweł Dubiel

pawel2.dubiel@gmail.com

1 Faculty of Geology, Geophysics and Environmental Protection, AGH University of Science and Technology, Kraków, Poland
- propagation of $\mathrm{S}$ waves only in the elastic medium, which means no impact of the fluid/gas on its propagation velocity;

- shear wave splitting phenomenon, which enables anisotropy analysis based on the $\mathrm{P}-\mathrm{SV}$ and $\mathrm{P}-\mathrm{SH}$ wavefields.

Due to these properties of $\mathrm{S}$ wave, the converted wave data can provide valuable information regarding lithology, lithofacial changes, correlations and verification of amplitude anomalies on PP sections (Myśliwiec 2004c). Converted wave recordings increase furthermore the reliability of the interpretation of AVO/AVA data and the use of joint inversion.

Despite the considerable usefulness of this type of solutions, converted wave recordings are still, due to significant costs, acquisition, processing and interpretation difficulties, relatively rarely used in industrial practice, while research based on $\mathrm{P}$ waves is still the main source of knowledge about the subsurface.

The aim of the research was to develop the optimal processing sequence which would increase the reliability of the seismic interpretation of three-component data. The analyzed data consist of standard PP records and non-standard PS components of the wavefield (vertical and horizontal: PS $_{x}$ and $\mathrm{PS}_{y}$, respectively). 


\section{Geological setting of the area}

The Chałupki Dębniańskie 2D-3C experimental profile was located in the Podkarpackie Province, in Leżajsk and Przeworsk districts (Fig. 1). It is a common agricultural area situated on the floodplain of the San and Wisłok rivers. The surface is mainly covered by wet meadows.
The east part of the Carpathian Foredeep is our research area. The following schematic geological model of the eastern part of the Carpathian Foredeep is presented in Fig. 1. The image shows vertical and lateral diversity of the main facial types of the Miocene deposits.

Two basic elements can be identified in the geological structure of this unit:

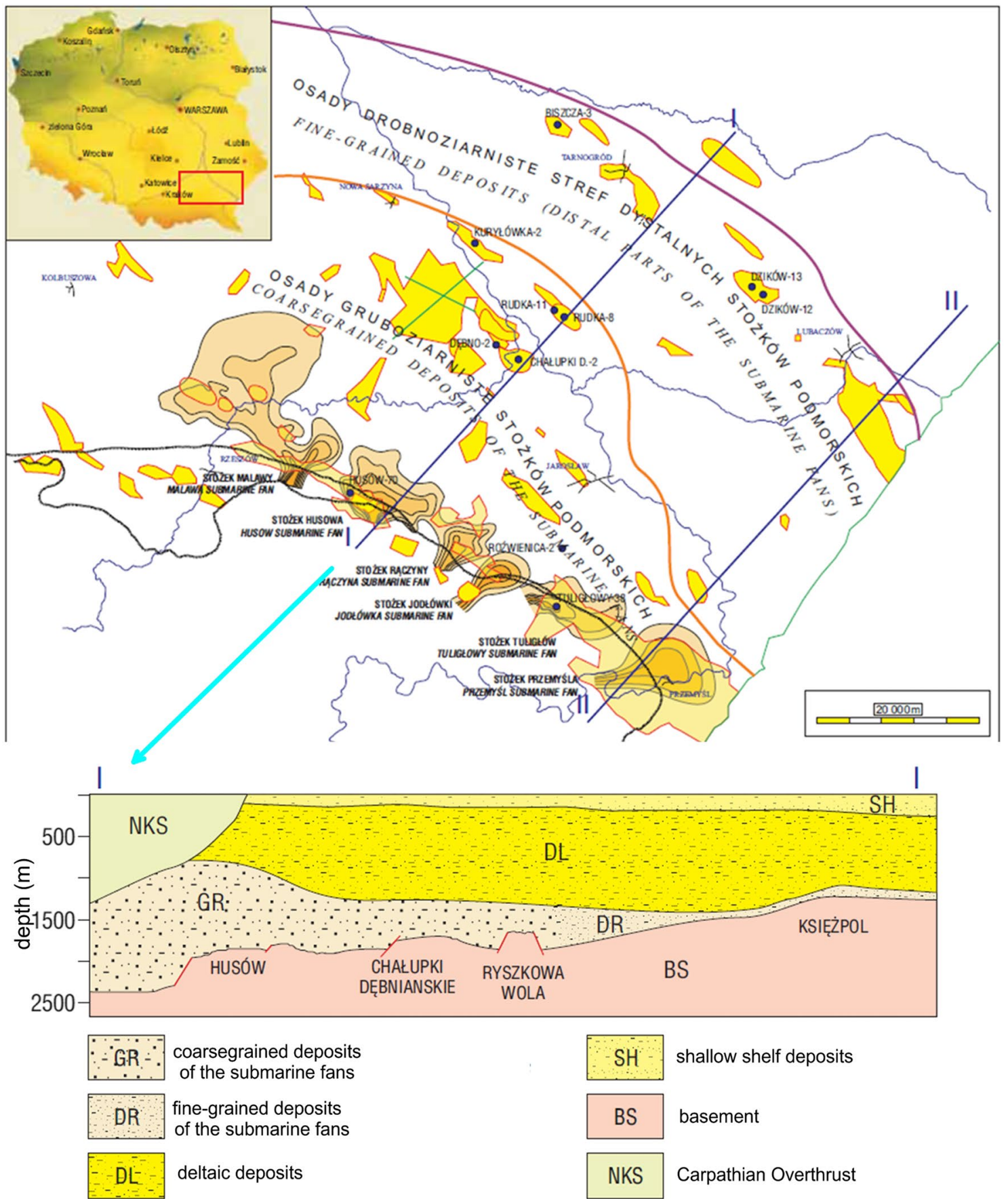

Fig. 1 Schematic cross sections through the eastern part of the Carpathian Foredeep—after Myśliwiec (2004b) 
- consolidated rocks lying on the bottom of the Miocene basin,

- neogeneous forms filling the Miocene basin.

In the research area, the basement is composed of shales interlayering of sandstones. In the regional scale tectonic forms, such as cracking and folding, are here very common.

Miocene is represented by the following sediments:

- Lower Badenian;

- Middle Badenian;

- Upper Badenian;

- Sarmatian.

On the Miocene formations, Quaternary sediments are present, such as clays, sands, gravels, locally loesses. The thickness of the sediments ranges from 5 to 25 meters.

Myśliwiec (2004c) describes traps for gas accumulations in the Miocene strata in the Carpathian Foredeep. Among them there is Chałupki Dębniańskie area with gas-bearing zones. In our research we have selected three perspective areas in terms of the hydrocarbon presence which will be analyzed. Two of them can be checked and verified by well log data. The third zone of interest is interpreted only on the basis of seismic data. Further, we will focus on the analysis of the selected zones.

\section{Data characterization}

The experimental seismic profile, which is the subject of this research, was part of the first multicomponent recording in Poland. The data were acquired in 2002. The purpose of the survey was (1) to develop the methodology for processing and interpretation of this type of recordings and (2) to apply the analysis results to the identification of natural gas deposits within the Miocene in the Carpathian Foredeep. First reports come from Gruszczyk et al. (2002). The data analyzed in the article is the 13D-4-02K seismic line, acquired with the dynamite source located in deep shot holes and VectorSeis digital geophones (three-component recording).

Basic acquisition parameters are presented in Table 1. The acquisition parameters allowed for the proper recording

Table 1 Seismic acquisition parameters

\begin{tabular}{ll}
\hline Source type & Dynamite, average uphole depth $=15 \mathrm{~m}$ \\
Receiver type & VectorSeis digital geophones \\
Shots & 305 shots with shot interval $=20 \mathrm{~m}$ \\
Receivers & 280 channels with receiver interval $=10 \mathrm{~m}$ \\
Offset range & $-2125-1410 \mathrm{~m}$ \\
Recording time & $5000 \mathrm{~ms}$ \\
Sample rate & $2 \mathrm{~ms}$ \\
Nominal fold & 60 \\
\hline
\end{tabular}

of PP and PS wavefields (in particular types of geophones and receiver spread long enough to receive converted wave). Locations of sources, receivers and values of nominal fold are presented in Fig. 2. It is noticeable that fold values are three times greater than nominal fold because the data consist of three components of the wavefield and they are stored in the same database. CDP fold is the important parameter controlling the quality of the final data. In this case fold values allowed good stacking quality.

\section{PP-wave processing}

The first and the most important research objective was to obtain the best resolution of seismic data (both prestack and stack image) with preserved relative amplitudes (RAP processing). Such a methodology enables interpretation of amplitude versus offset (AVO) and amplitude versus incidence angle (AVA). AVO anomaly, which is visible on prestack seismic gathers on times representing the Miocene strata, results from hydrocarbon saturation observable in this area. AVO/AVA processing aims are: (1) to estimate and calculate the signal energy losses during propagation in the medium, (2) to remove the impact of noise and the weathering layer, and (3) to remove processing-related artifacts (Chopra and Castagna 2014). This assumption requires a careful selection of procedures and a thorough quality control of the results.

Schematic processing flow of PP-wave recording used to obtain the final PSTM-migrated stack is presented in Fig. 3. The processes used in the sequence are the subjective choices of the authors and are common for PP-wave processing with relative amplitudes preservation. The first stage included database processing, seismic data import, binning and database QC. Afterward, preprocessing consisted of: bad trace killing (based on statistics of traces), despiking (removal of single sample spikes and multiple sample bursts of noise), bandpass filtering (minimum-phase 4-8-120-160 Hz Ormsby filter used basically for random noise attenuation) and spherical divergence compensation. Ground roll was the main source of noise, which covered lower parts of the recordings (below the target area). We therefore incorporated Surface Wave Noise Attenuation (process suitable for ground roll elimination, based on velocity and frequency content of the noise). The same process was used for direct wave attenuation. Surface consistent amplitude correction and deconvolution were executed after elevation and then refraction statics calculation and application. Velocity analysis and residual statics (calculated by maximizing the CDP stack power) were performed in two iterations. Figure 4 shows brute stack (Fig. 4a) and the stack before migration (Fig. 4b). The difference between images is substantial, which testifies that the applied processes allowed better imaging of seismic boundaries. 


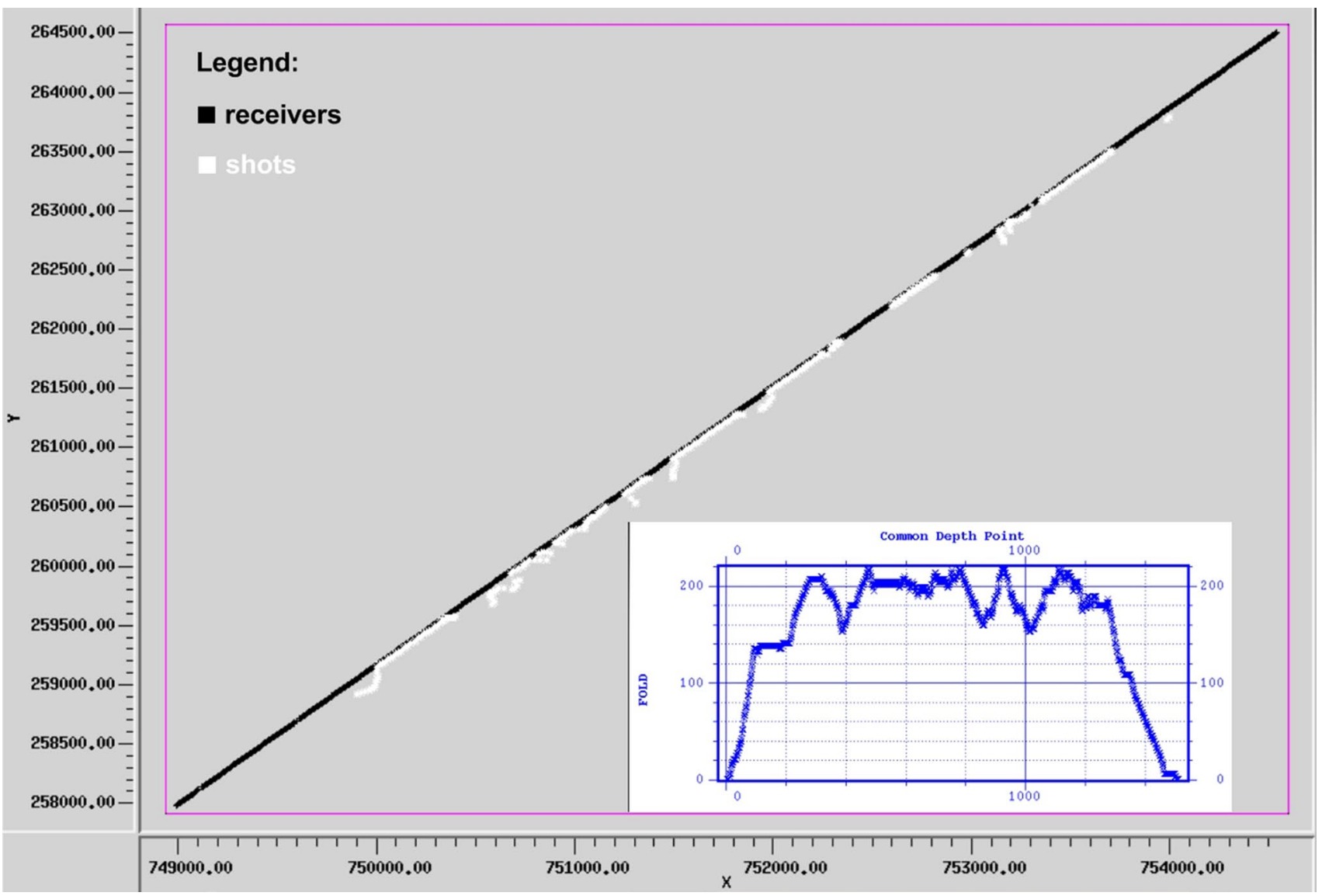

Fig. 2 Shot/receiver basemap and the CDP fold plot for the entire line

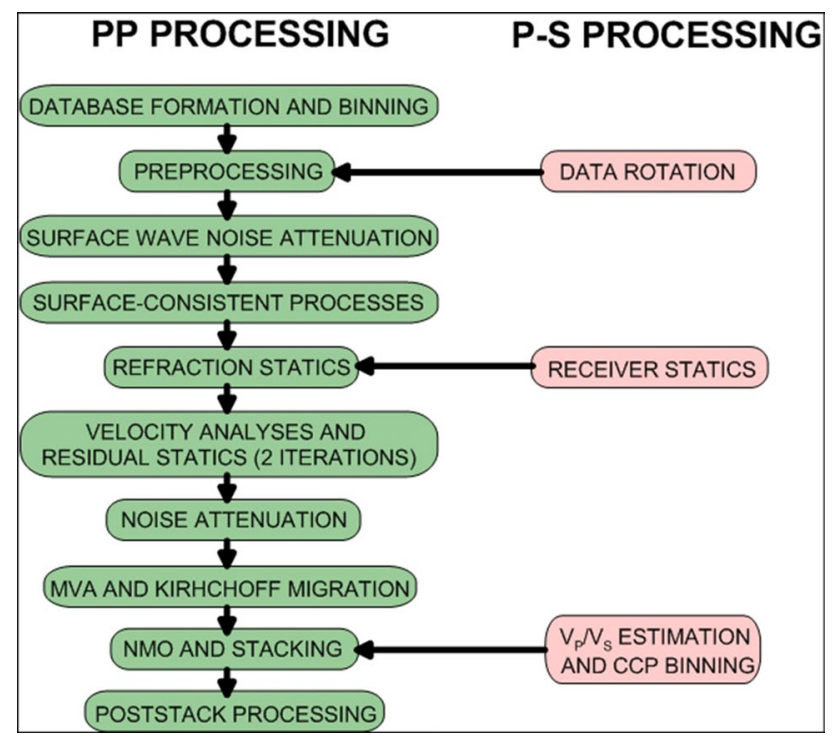

Fig. 3 Processing sequence designed by the authors. Processes used for PP-wave data recordings (on the left) and additional tools used for PS-wave data (on the right)
The analyzed data were migrated with Kirchhoff algorithm to be suitable for AVO study. This is the most computationally demanding solution (e.g., Yilmaz 2001). An unquestionable advantage of the chosen method is the ability to perform migration velocity analysis (MVA). Another possibility is the direct migration of the calculated AVO attributes (Chopra and Castagna 2014).

Stacking velocity field, which was used in migration velocity analysis, was initially smoothed (Fig. 5). It is visible that on time between 200 and $500 \mathrm{~ms}$ the velocity in the central part of the profile is lower than on both sides of the profile (Fig. 5). It is related to the main reservoir existing in Chałupki Dębniańskie area. Thus, the central reservoir seems to be large and rich in gas, big enough to cause the P-wave velocity decrease.

Poststack processing included spectral whitening, timevariant frequency filter and FX deconvolution (Treitel 1974). It was directed at signal-to-noise ratio improvement. 

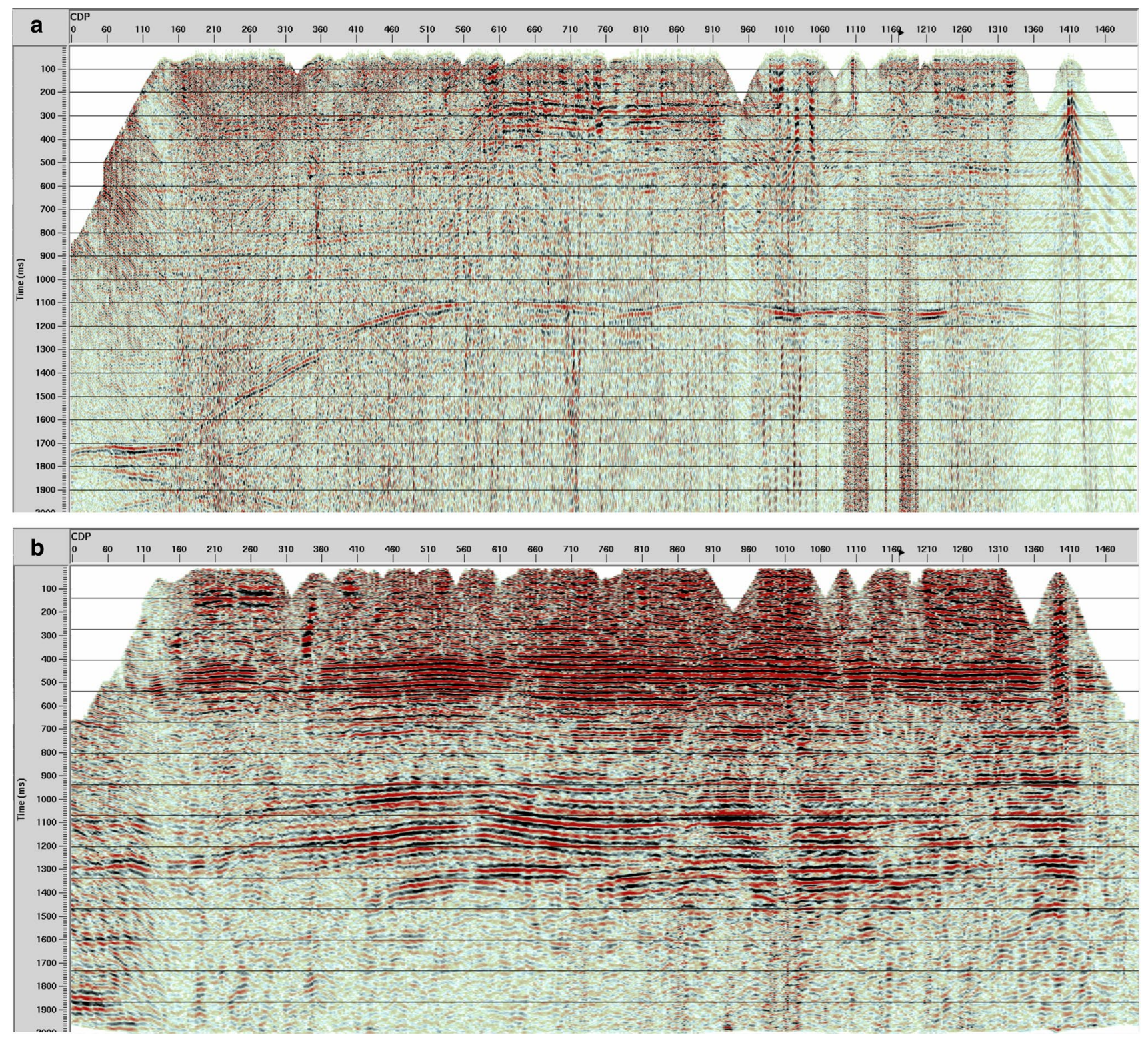

Fig. 4 Comparison of a brute stack (a) and the stack before migration (b)

\section{PS-wave processing}

Converted wave recordings have much lower data quality than PP-wave recordings due to the behavior of the reflected $S$ wave. Their processing is difficult and demanding, especially when it is directed at relative amplitude preservation. The final result of two acquired PS components (PS $x$ and $\mathrm{PS}_{y}$ ) will be compared to the PP data in order to find the differences in seismic images related to the gas occurrence.

Most of the procedures used (attenuation of coherent and random noise, deconvolution) were processed in the same manner and in the same order as for the PP component (Fig. 3), differing only in parameters such as the size of the time gates or the filter frequencies. The fundamental differences are related to the specific properties of converted waves, in particular: (1) data rotation (2) estimation of the receiver statics and (3) estimation of $V_{\mathrm{P}} / V_{\mathrm{S}}$ and common conversion point binning (Stewart et al. 1999). Procedures essential for PS components processing sequence and their location in the workflow are shown in Fig. 3. In this chapter we will discuss only these specific differences. 


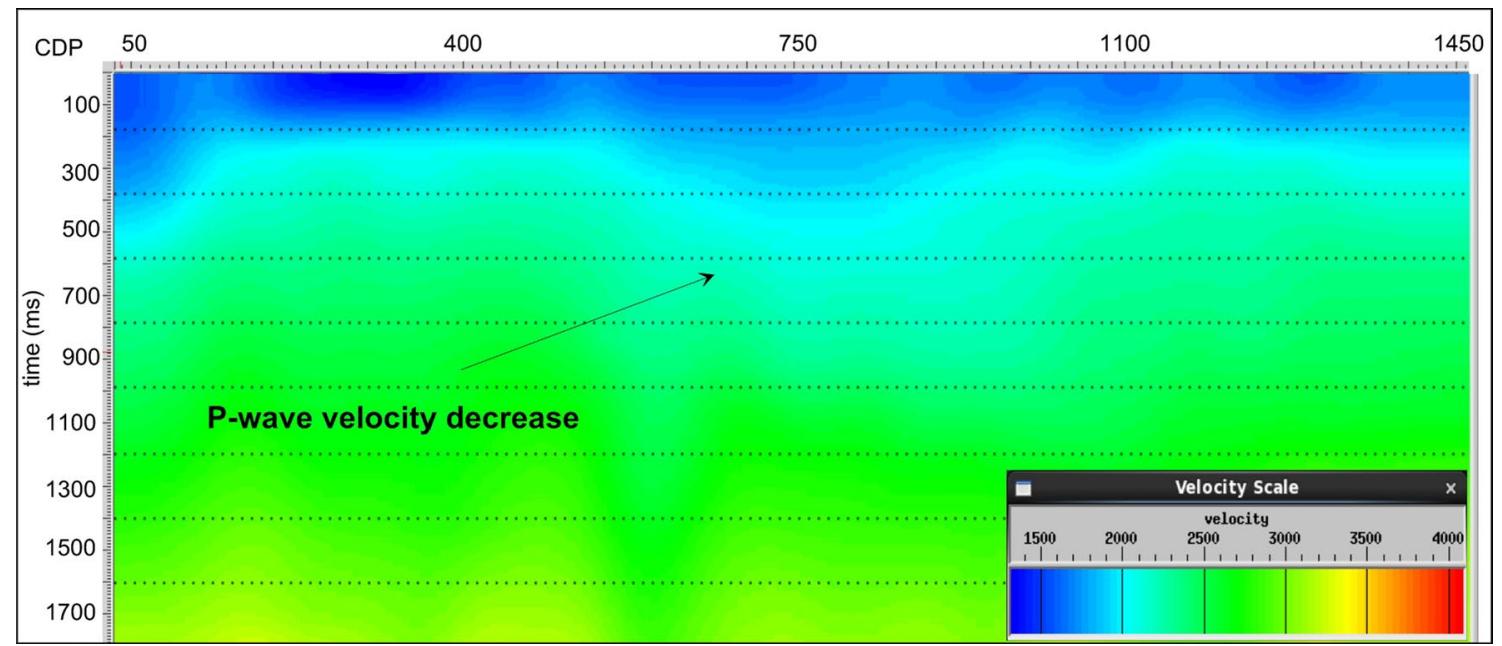

Fig. 5 Final smoothed stacking velocity field of $\mathrm{P}$ wave. In the central part of the profile, values of the P-wave velocity are lower due to the presence of gas-bearing layers in the Chałupki Dębniańskie area

\section{Data rotation}

In case of isotropic medium, most of the converted wave energy is recorded on the radial component (in-line direction), while on the cross-line component the white noise should be recorded (Jezierska and Keller-Utracka 2003). Despite the proper orientation of the geophones on the profile, it is usually impossible to place the source exactly in the line of the receiver points, so it is necessary to rotate the recorded data from the physical measurement system to the radial and transverse system. As a result, a change in the polarization of the negative offsets and the improvement of the continuity of shallow horizons on the seismic section can be obtained.

\section{Receiver refraction statics}

Refraction statics for receiver, in case of converted waves, is a problematic issue due to high variability of S-wave velocity and strong attenuation in low velocity layer. Standard approach is based on first break picking, but very often first breaks have very low quality. Residual statics algorithms often fail because of large static shifts and cycle skipping. To determine statics for receivers, common receiver stacks were used. This approach was discussed by Dufour (1996). At first the full shot and receiver refraction statics (obtained during PP-wave recordings processing) was applied to the PS section. On PS section one strong and clear horizon was picked to retain dip information. Next seismic section was smoothed and correlated with previous stack. The differences between both images were calculated by iterative processes. On this basis the receiver statics was estimated and applied to the PS recordings (Fig. 6). Refraction statics application yielded the significant improvement of the horizons continuity (especially in places marked with arrows in Fig. 6).

\section{$V_{\mathrm{P}} / V_{\mathrm{S}}$ estimation and CCP binning}

As a result of velocity analysis, $V_{\mathrm{S}}$ velocity was estimated and smoothed (Fig. 7). It can be seen that in the central part of the seismic profile (the main reservoir) $V_{\mathrm{S}}$ is almost not decreasing in contrast to Fig. 5. It indicates directly that $\mathrm{S}$ wave is not sensitive to the gas saturation. Other perspective areas do not cause the velocity decrease in Figs. 5 and 7 , which can be the first important clue about hydrocarbon saturation level.

$V_{\mathrm{P}} / V_{\mathrm{S}}$ ratio (marked as $\gamma$ ) is the crucial parameter during converted wave processing. It is responsible for determining the common conversion point (CCP). The use of an incorrect value of this parameter may lead to false positioning of seismic boundaries and, in general, degradation of the stack quality. The standard method for $\gamma$ estimation is the correlation of the same seismic boundaries on the migrated PP and PS stacks and calculation of the interval $V_{\mathrm{P}} / V_{\mathrm{S}}$ ratio $\left(\gamma_{0}\right)$ or the gamma scan usage, similar to velocity analyzes (Mancini et al. 2002). Another method is using of the $\gamma_{\mathrm{NMO}}$ parameter, which is the ratio of the stacking $V_{\mathrm{P}}$ and $V_{\mathrm{S}}$ velocities. The second method (presented in Fig. 8) was used in the research. $\gamma_{\mathrm{NMO}}$ values range from 1.9 for shallow reflections (TWT 0-500 ms) to 1.3 for deeper reflections (TWT $1500-1800 \mathrm{~ms})$. It can be seen that in deeper layers $\gamma_{\mathrm{NMO}}$ is generally smaller than in shallow layers, which is the result of greater compaction. 


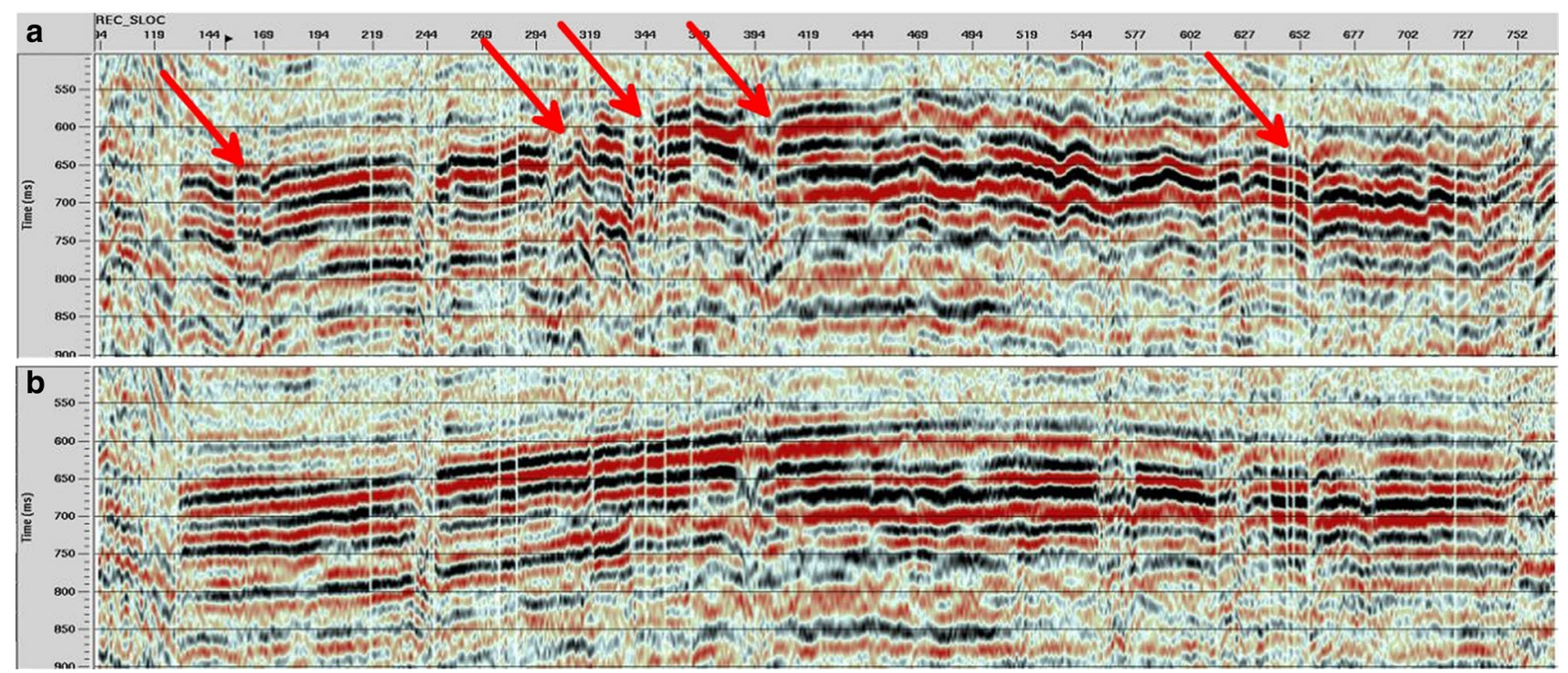

Fig. 6 Common receiver stack (a) before refraction statics and (b) after refraction statics (source statics based on PP-wave recordings and estimated receiver statics), after Dubiel (2018)—modified

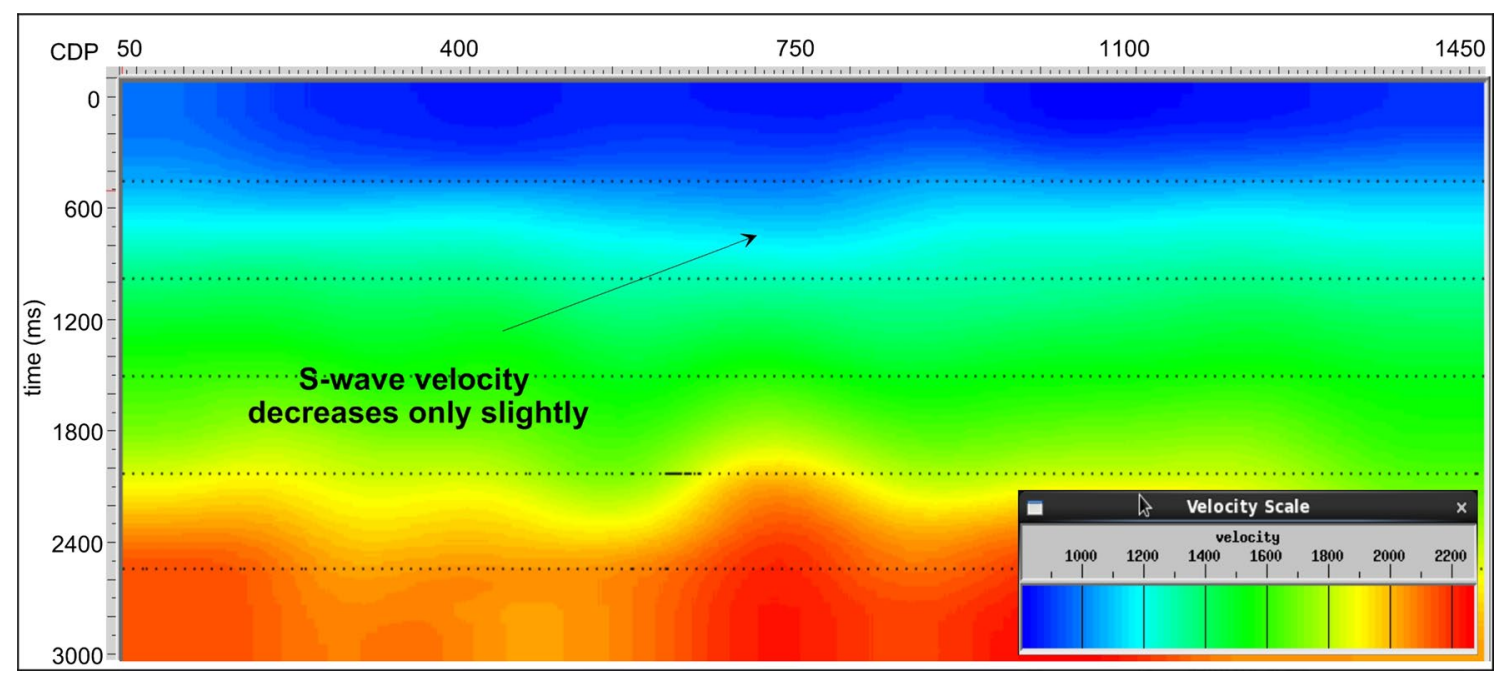

Fig. 7 Final smoothed stacking velocity field of S wave. In the central part of the profile in the area of the hydrocarbon reservoir, S-wave velocity decreases very slightly

\section{$3 C$ seismic results}

For all components (PP, $\mathrm{PS}_{x}$ and $\mathrm{PS}_{y}$ ), two types of datasets were obtained: (1) prestack migrated gathers (suitable for AVO analysis) and (2) poststack migrated sections (appropriate for seismic attributes calculation). All of the datasets were used in the interpretation.

In all cases (PP, $\mathrm{PS}_{x}$ and $\mathrm{PS}_{y}$ ) two types of poststack data were generated. The first set of data had preserved amplitude relations (RAP processing on PP, $\mathrm{PS}_{x}$ and PS $_{y}$-Figs. 9a, 10a and 11a, respectively). On the second dataset, AGC scaling and ampltude balancing was applied
(AGC processing on PP, $\mathrm{PS}_{x}$ and $\mathrm{PS}_{\mathrm{y}}$-Figs. 9b, 10b and $11 \mathrm{~b}$, respectively). The final PreSTM PP seismic sections (Fig. 9) show large differences in amplitude level, thus revealing the areas of high acoustic impedance contrasts. They are most visible on 600-1000 CDP range within 100-500-ms time interval. Another anomalous amplitude of horizons is observed on 1200-1300 CDP range within 400-800-ms time interval. In this zone many layers are on high amplitude levels, which can suggest gas existence. Strong reflection is also associated with the horizon at time $1000 \mathrm{~ms}$. 


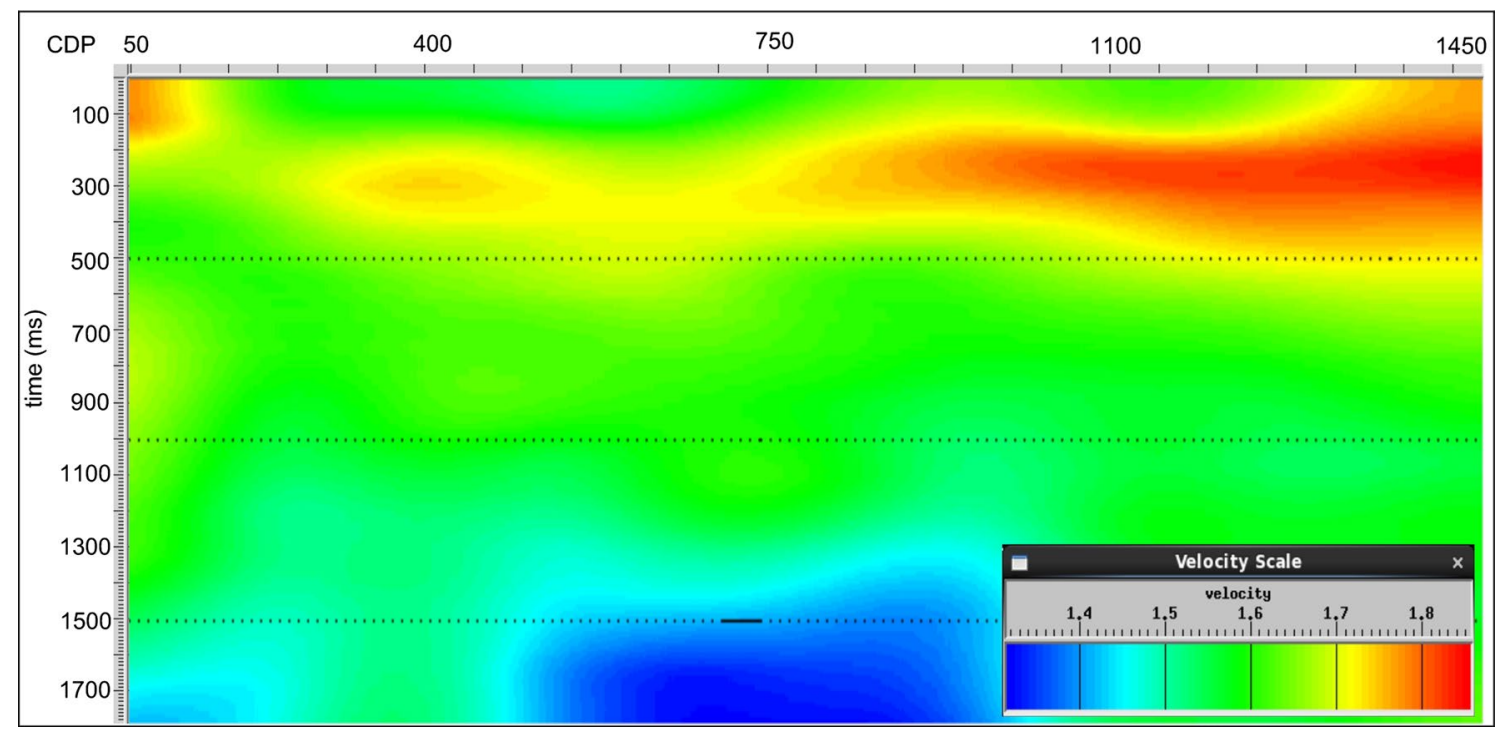

Fig. $8 \gamma_{\mathrm{NMO}}$ distribution shown in PP-wave $t_{0}$ time

On the other hand, when we compare final PreSTM PS seismic sections in RAP version (Figs. 10a, 11a), we can distinguish horizons of high amplitude levels but there are no isolated areas characterized by strong acoustic impedance contrast. Since $\mathrm{S}$ wave propagates only in solids, it is insensitive to the gas/fluid existence. However, some boundaries characterized by a very strong acoustic impedance contrast, as the one observed on $1100 \mathrm{~ms}$ in Figs. 9 and 12, are less visible on PS components (Figs. 10, 11, 12b, c). This fact results probably from the low quality of the PS recordings on later TWT. Furthermore, on all PS sections (Figs. 10, 11) amplitude differences between RAP and AGC processed data were much smaller. On the basis of this observation, we stated that the PP wave is much more sensitive to amplitude variations due to the hydrocarbon saturation than $S$ wave.

At the end of the processing stage, we discovered that the resolution and general quality of PS data is lower than $\mathrm{PP}$ data. The reason for that is the stronger attenuation of $\mathrm{S}$ wave, as mentioned before. Also, because the S-wave velocity is much smaller than P-wave velocity (see Figs. 5, 7), we are not able to compare the corresponding reflections on PP and PS datasets. Therefore, in the next chapter we present and discuss PS sections transformed to the $\mathrm{t}_{0}$ time of PP wave.

\section{Basic interpretation of the seismic data}

Basic interpretation of the data consists of the well log and PP and PS poststack data correlation. In Fig. 12 three seismic sections are presented ( $\mathrm{PP}, \mathrm{PS}_{x}$ and $\mathrm{PS}_{y}$, all in RAP version).
$\mathrm{PS}_{x}$ and $\mathrm{PS}_{y}$ sections were recalculated using the estimated $V_{\mathrm{P}} / V_{\mathrm{S}}$ to allow more reliable seismic interpretation.

In direct vicinity of seismic line, two boreholes are located (CHD-2 around CMP 770 and CHD-3 around CMP 1170). Both are reaching the top of the Precambrian (Fig. 12a). On PP seismic section, selected well log data were presented: water saturation (green curve), gamma ray (black curve) and certain top formations (M0, MI, MII and Anh(st)). In the wells basic logs are available (GR, RHOB, DT, VSH, NPHI, SW and SP), but the lack of experience in multicomponent data interpretation resulted in no measurement of the interval time of the $\mathrm{S}$ wave for the entire crosssection. Marzec et al. (2006) proposed method of calibration of measured or synthetic $\mathrm{S}$-wave curves on the basis of the $\mathrm{P}$-wave distribution and recorded $\mathrm{P}$ - and $\mathrm{S}$-wavefields. To limit some uncertainties during seismic and well log data interpretation, we were focused on the proper S-wave velocity field estimation at the processing stage.

Among the seismic attributes, which are used as hydrocarbon indicators, we consider bright spots and time sags. Qualitative interpretation is based on the fundamental property of $\mathrm{S}$ wave, mentioned at the beginning of the work, i.e., hydrocarbon saturation has no effect on the S-wave velocity. This enables the verification of amplitude anomalies present on the PP section.

According to the analysis of PP sum, three bright spot zones were selected. (They reveal large differences in acoustic impedance.) The areas are located inside red rectangles and numbered in Fig. 12. Bright spots, resulting from large differences in acoustic impedance at the top and bottom of the saturated layer, are a frequent phenomenon in the Miocene deposits in the Carpathian Foredeep (Myśliwiec 2004a, 


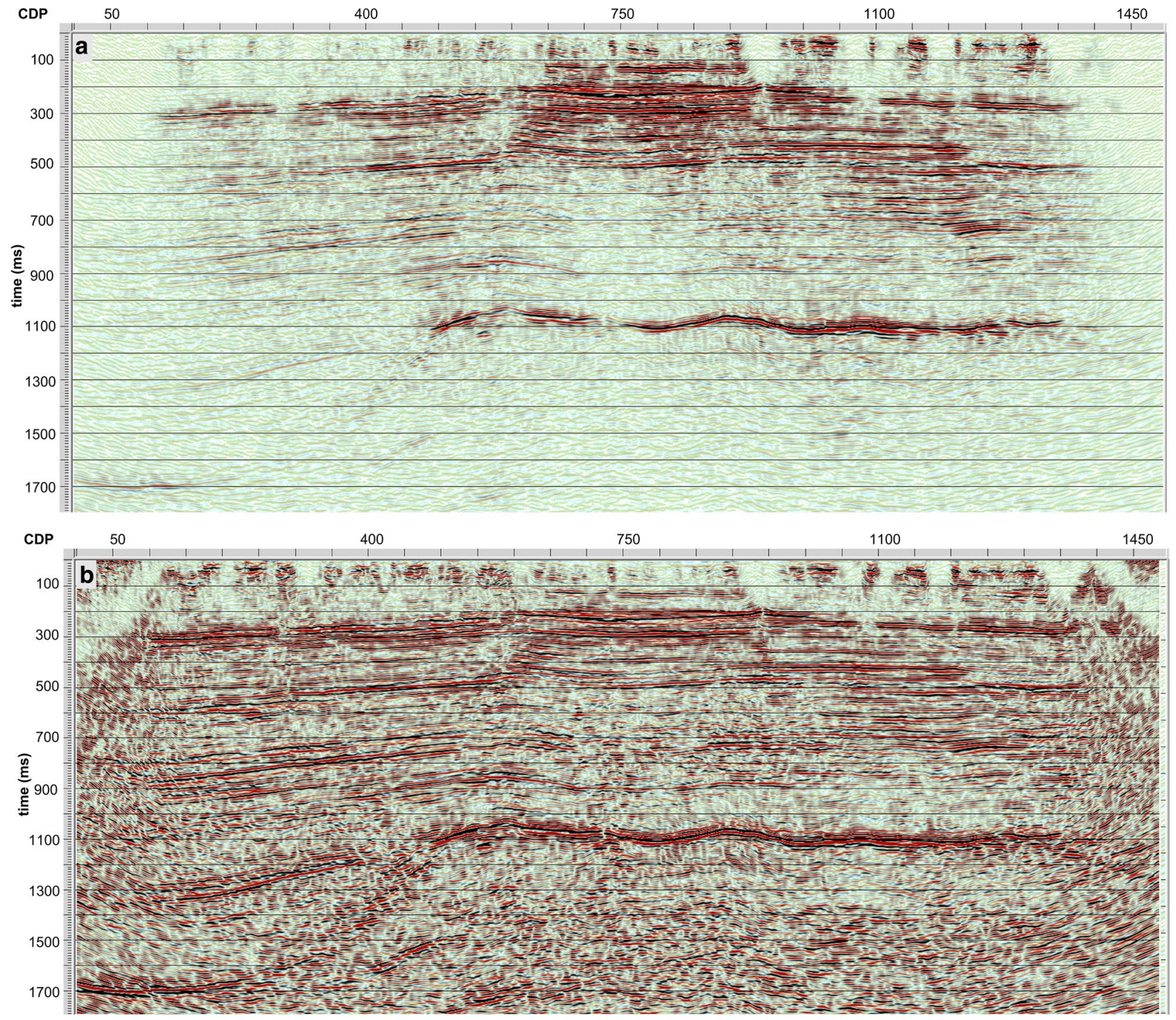

Fig. 9 Final PreSTM PP seismic section with poststack processing: a RAP version, b AGC version

2004c). The basic problem of the PP sections interpretation is the fact that other factors, such as lithology, can cause similar amplitude effects. However, an anomaly, which is the result of lithology change, shall be recorded on all wavefield components, while anomaly resulting from hydrocarbon saturation will be recorded only on PP wavefield image (only in RAP version). Unfortunately, the small gas saturation can also induce large amplitude anomaly (Chopra and Castagna 2014).

The CHD-2 well is located in the main reservoir area on the seismic section (zone number 2). There is a strong correlation between decreased water saturation (green curve in Fig. 12a) and the increased amplitudes of seismic horizons in time interval of 250-500 ms on PP section. From well $\log$ data it can be concluded that the reservoir is rich in organic matter and has good reservoir properties (17 gasbearing layers have been detected); therefore, the correlation between seismic and well log data is evident and easy for interpretation.

The CHD-3 passes through the third reservoir area (zone 3: CDP range 1100-1300, TWT: $500-800 \mathrm{~ms}$ in Fig. 12). Lower values of water saturation are detected almost in the whole depth interval. The performed studies suggested that this zone is in fact non-productive. Myśliwiec (2004a, 2004c) and Chopra and Castagna (2014) explain that the strong amplitude anomaly may be also caused by the existence of many layers saturated by a small amount of gas or by bad reservoir properties. During drilling hydrocarbon saturation was not taken into account and no additional measurements were taken. Therefore, the correlation of seismic and 


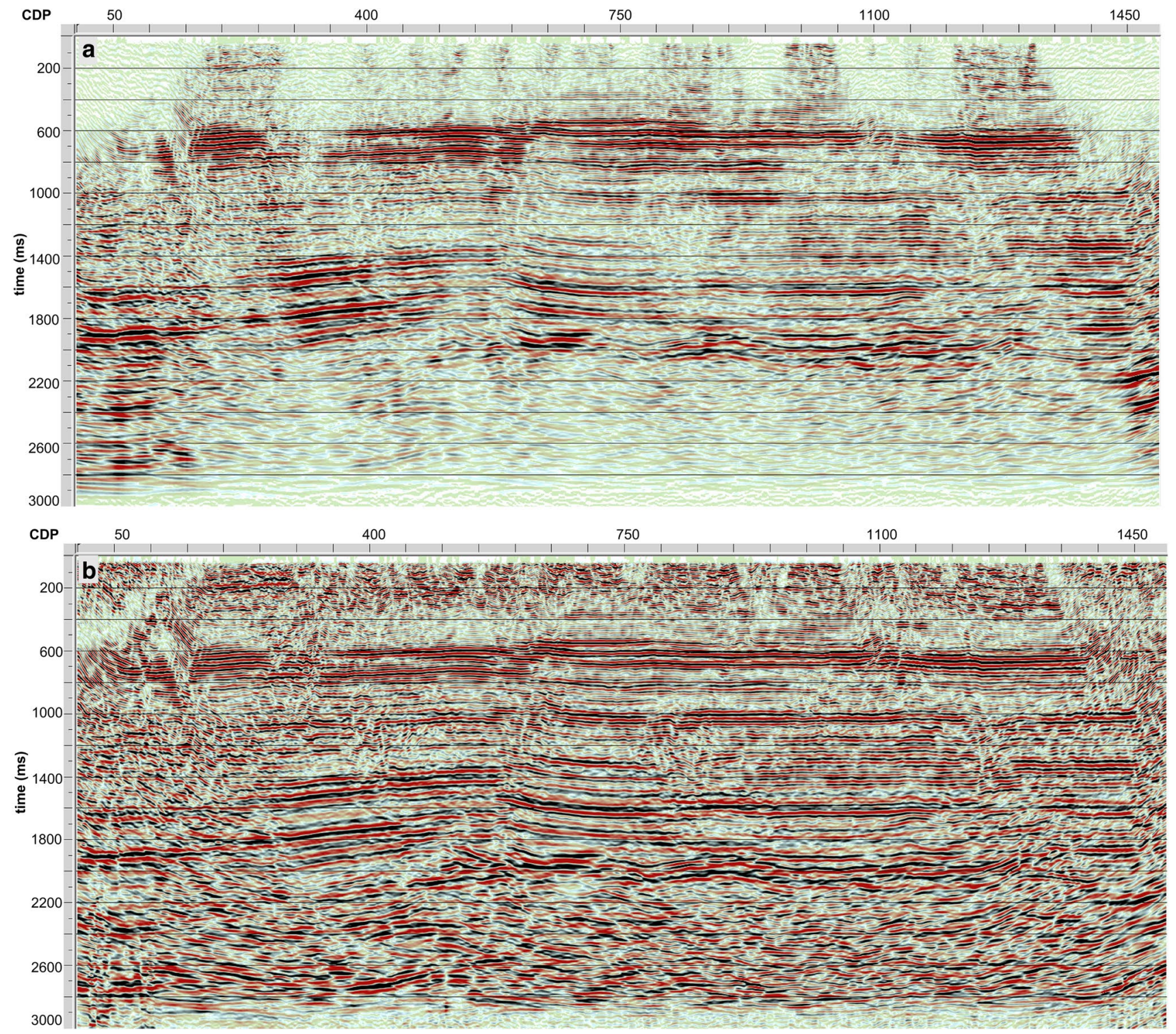

Fig. 10 Final PreSTM PS $x$ seismic section: a RAP version, b AGC version

well log data is evident, but the analysis of reservoir properties is very doubtful.

$\mathrm{PS}_{x}$ and $\mathrm{PS}_{y}$ sections in RAP version (Fig. 12b, c) give additional information about potential gas accumulations. Amplitudes of the horizons in red boxes in Fig. 12c are also increased, but their anomalous amplitude behavior is detectable almost on the whole profile. This suggests the impact of the lithology on reflection coefficient on the particular boundaries. Inversely, on the $\mathrm{PS}_{x}$ section horizons are very weak except the ones on time interval 150-300 ms, but still their amplitude is comparable on the whole section. When comparing PP and PS images (Fig. 12), it can be concluded that the local amplitude anomalies within selected zones occur only on PP sum. This fact suggests that they are the result of hydrocarbon saturation.
The statistical wavelet extracted from seismic section was used for well logs tying. On PP and PS data, three horizons within Miocene and top of the Precambrian were correlated (denoted in Fig. 13 as hor0, hor1, hor2 and hor3, respectively). Main difficulty regarding well-to-PS seismic sections tying was the use of correct $\mathrm{S}$-wave velocity, which was interpreted during processing.

In Fig. 13 we present selected well log data for CHD-2 and corresponding seismic data, in particular: GR, SP, RHOB, DT, PHI and SW, part of seismic section near CHD-2 and the nearest CDP gather. Gas zones are located between horizon 0 and horizon 1 (marked as hor0 and hor 1 in Fig. 13, respectively). There is a clear correlation between water saturation (SW) and the level of seismic amplitude in direct well area. Much lower values of water saturation 


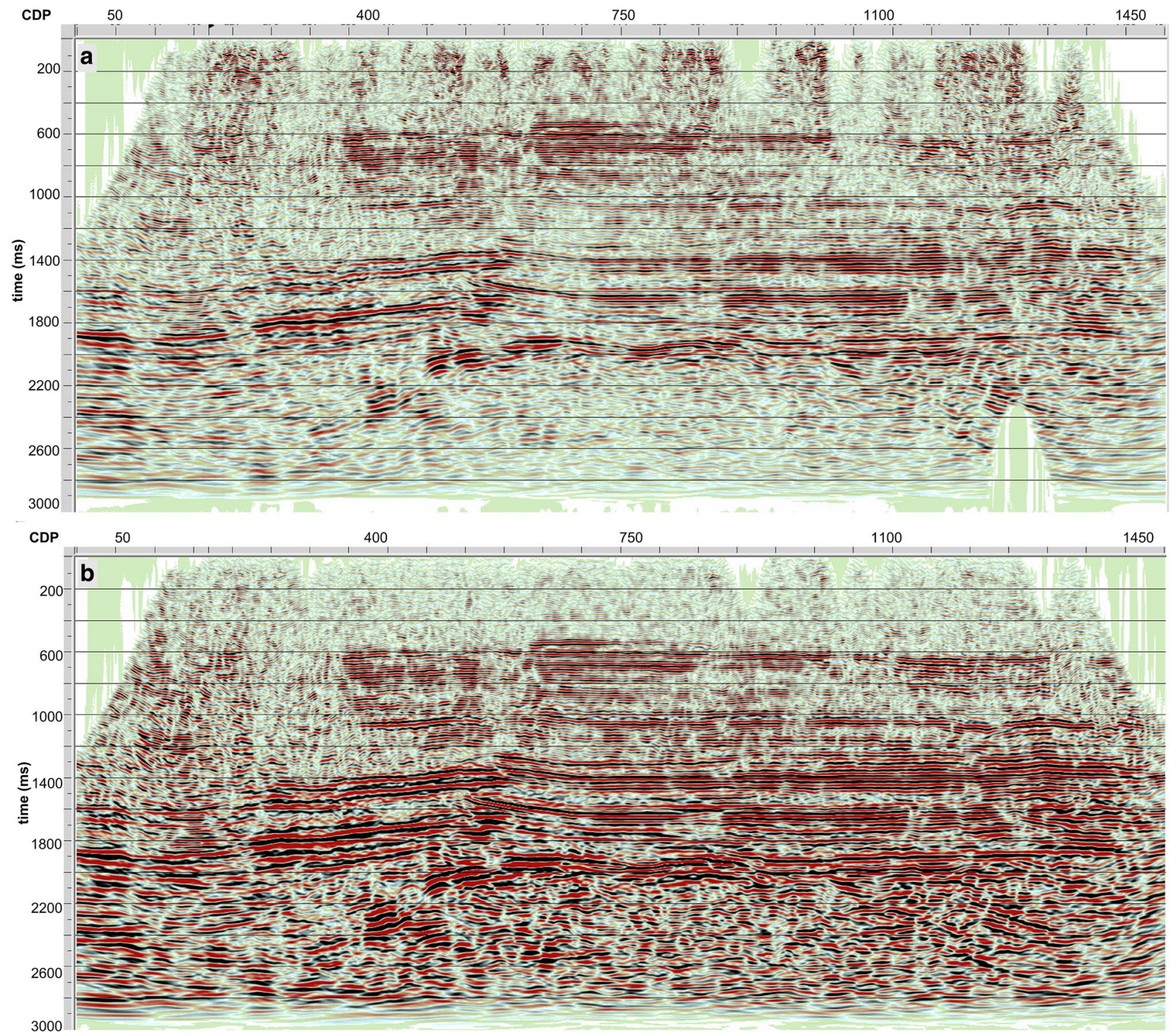

Fig. 11 Final PreSTM PS seismic section: a RAP version, b AGC version

correspond to higher amplitudes on seismic. Unfortunately, some measurements were not done from the surface; therefore, the interpretation of shallow part of the reservoir is limited.

Another DHI, frequently occurring in the Miocene strata of the Carpathian Foredeep, is so-called time sag (Myśliwiec 2004a). It is the effect of time mispositioning of boundaries within and under the gas-saturated zone, also associated with the reduction of $\mathrm{P}$-wave velocity. As the $\mathrm{P}$-wave velocity decreases in hydrocarbon saturated zone (Fig. 14a), seismic boundaries within and under the gas-saturated zone are placed below their correct position in time domain. On the $\mathrm{PS}_{y}$ section (Fig. 14b) time sag does not occur, because the $\mathrm{S}$-wave velocity is insensitive to the hydrocarbon saturation. Moreover, using PS data during interpretation enables better mapping of seismic boundaries under the reservoir, where a significant decrease in amplitude is observed due to the high impedance contrasts on the layer boundaries.

\section{AVO/AVA analysis}

Amplitude versus offset (AVO) study for $\mathrm{P}$ wave is based on the analysis of the reflection coefficient (RC) change with source-receiver offset. Similarly, amplitude versus angle (AVA) reveals the change of $\mathrm{RC}$ with an incidence angle. Both methods are commonly used in detection and estimation of the anomalous density and velocity contrasts for $\mathrm{P}$ wave along a given seismic boundary (Chopra and Castagna 2014). In fact, the AVO/AVA interpretation cannot be the only method used to verify the gas existence, because 


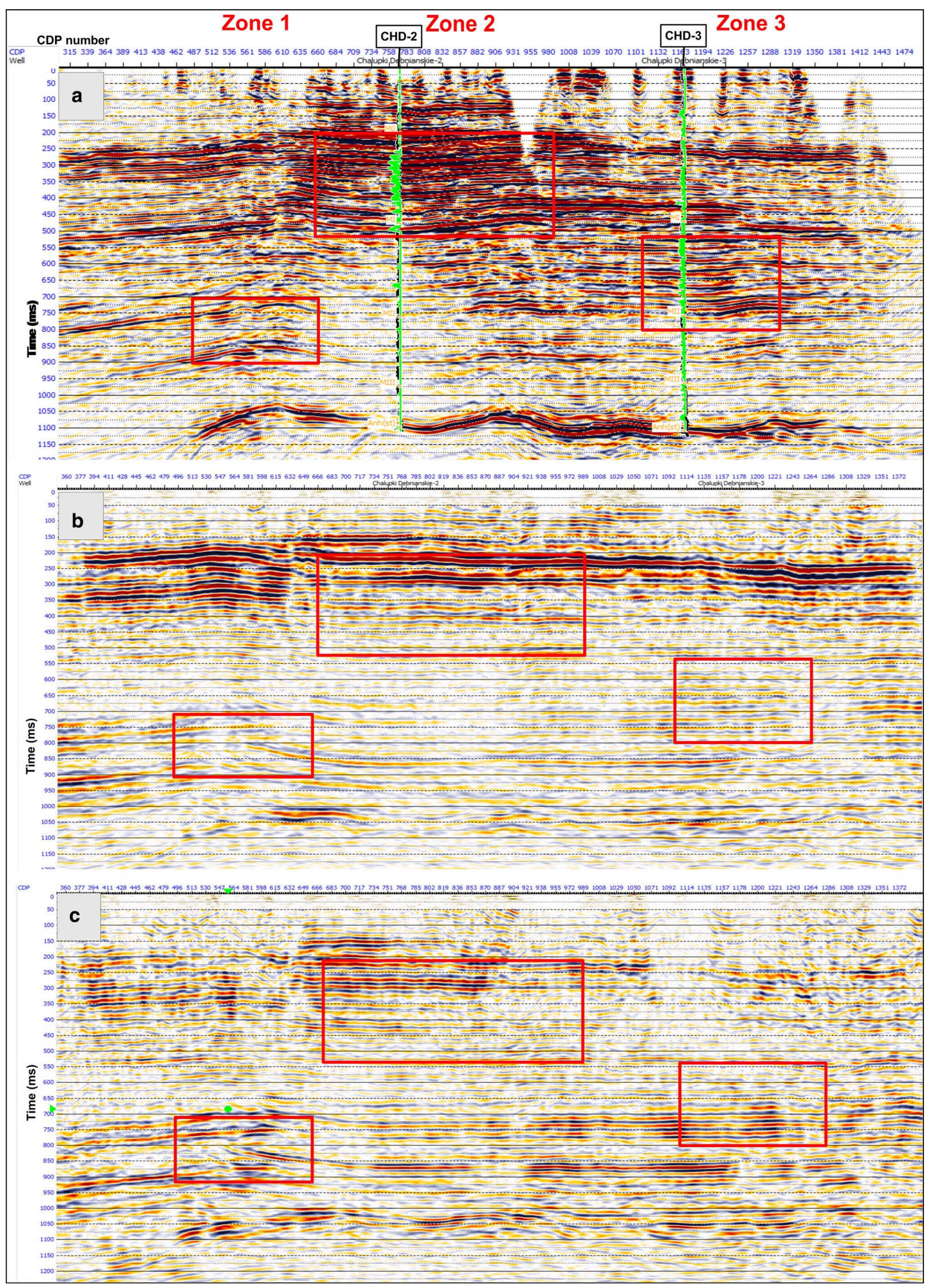


4Fig. 12 Comparison of a PP seismic section, b PS $_{x}$-wave seismic section and $\mathbf{c} \mathrm{PS}_{y}$-wave seismic section (all in time of PP wave). Two wells are shown (CHD-2 and CHD-3). Potential gas accumulations are located in red boxes and numbered

low-saturated layers, for example gas sands, can cause similar amplitude anomaly to those with high gas saturation (Chopra and Castagna 2014). There are many interpretation techniques using the described wave phenomenon, based on gather analysis (offset/angle gathers), sum analysis (offset/ angle limited stacks) and AVO/AVA attribute distributions (including Intercept and Gradient). Recently, there is greater interest in PP- and PS-wave joint AVO inversion, but the methodology is still rather rare (Gaiser 2016). These limitations can be the result of high data quality requirements.

AVO/AVA analyses are more demanding comparing to the bright spot interpretation. A case study is conducted on prestack data (lower $\mathrm{S} / \mathrm{N}$ ), and it is necessary to determine and remove the influence of acquisition and wave propagation on the amplitude of the signal. This means much more careful and demanding processing of seismic records. In order to improve signal-to-noise ratio and coherence of the reflections, additional processing was conducted. In this research we focused only on the interpretation of PP-wave AVA anomaly, because we did not obtain the satisfactory quality of the PS data.

AVA analysis was performed in all zones (1, 2 and 3). In the vicinity of the zone 1 , prestack data do not reveal change of amplitude with offset; therefore, AVO/AVA analysis for this zone will not be discussed in the paper. The main reservoir area (zone 2) generates a classical and easy to interpret anomaly which was widely discussed in previous studies (for example Dubiel 2018). In this paper we show the results of AVA analysis for zone 3 (Figs. 15, 16).

The angle gathers were created on prestack CRP gathers (Fig. 15). A range of angles from 1 to $45^{\circ}$ with an interval of $2^{\circ}$ was used, obtaining 22 traces for each CMP. This range of angles was necessary to obtain the same effective coverage for the entire time interval of interest as for CMP gathers. Four angle gathers were generated in the range of: (1) $1-22^{\circ}$ (2) $11-33^{\circ}$ (3) $22-44^{\circ}$ and (4) in the whole range of angles: $1-44^{\circ}$. Presented angle gathers show amplitude increase with angle in the specified time interval (Fig. 15b).

AVA analysis was performed for the top and bottom reflection within 700-750 ms time interval (Fig. 16). Having the reflection amplitude as a function of the square of the sine of the incidence angle, we could calculate the following AVO attributes: Intercept and Gradient (denoted as $P$ and $G$, respectively). Crossplot of the Intercept, calculated as the reflection coefficient at zero angle of incidence, and Gradient, representing the slope of the line on the aforementioned plot, are shown in Fig. 16c.
Amplitude versus offset plot for top and bottom reflection (Fig. 16b) and crossplot of Intercept and Gradient (Fig. 16c) suggest that in this case we are dealing with the third class of AVO, which is typical for poorly consolidated sandstones saturated with gas and surrounded by rocks of much higher impedance. Unfortunately, measurable AVO anomalies can also be generated by a relatively low level of gas saturation. A standard analysis of PP-wave recordings is not sufficient to distinguish the degree of saturation (Myśliwiec 2004c).

Angle gathers were used to create the stacks of selected AVO attributes (Fig. 17). Intercept $P$, Gradient $G$ and their combinations (in particular product of $P$ and $G$, named as Product) are typical hydrocarbon indicators. In the analyzed case we are dealing with third AVO class, where sandstones saturated with gas have lower acoustic impedance than surrounding rocks. This results in negative value of the Intercept and negative Gradient (therefore Product will be positive-Fig. 17c) (Chopra and Castagna 2014). Another attribute used in the analysis was Scaled Poisson Ratio Change (Fig. 17d). Abrupt change in the Poisson's ratio results in negative reflection coefficient from the top of the saturated layer and positive reflection coefficient from the bottom of the saturated layer. For computation the two-term Aki-Richards approximation was used (Aki and Richards 1980). The range of incidence angles was $2-35^{\circ}$. It is evident that all presented AVO attributes show high amplitude variations in zones 2 and 3 , while zone 1 does not reveal anomalous amplitudes. This confirms previous statement that the amplitude changes visible on PP recordings within zone 1 are not the result of hydrocarbon saturation.

\section{Conclusions}

The paper confirms that multicomponent seismic significantly supports seismic data interpretation due to the differences in S-wave propagation. Good example for that is a direct hydrocarbon indicators verification, such as bright spots and time sags. Another advantage is the possibility of using prestack seismic data for AVO/AVA analysis.

On the other hand, three-component seismic processing is demanding and difficult. The significant problems are the data rotation, estimation of the receiver statics and reliable estimation of $V_{\mathrm{P}} / V_{\mathrm{S}}$ ratio used for CCP binning and the transformation of $\mathrm{S}$-wave recording to the time of PP-wave recording. In the research no additional assumptions regarding anisotropy were formulated; however, when analyzing the sums of $\mathrm{PS}_{x}$ and $\mathrm{PS}_{y}$, it can be certainly stated that anisotropy occurs in the analyzed area and is associated not only with the presence of cracks, fissures and faults, but also with the variability of rock layering and granulation (Myśliwiec 2004a). 

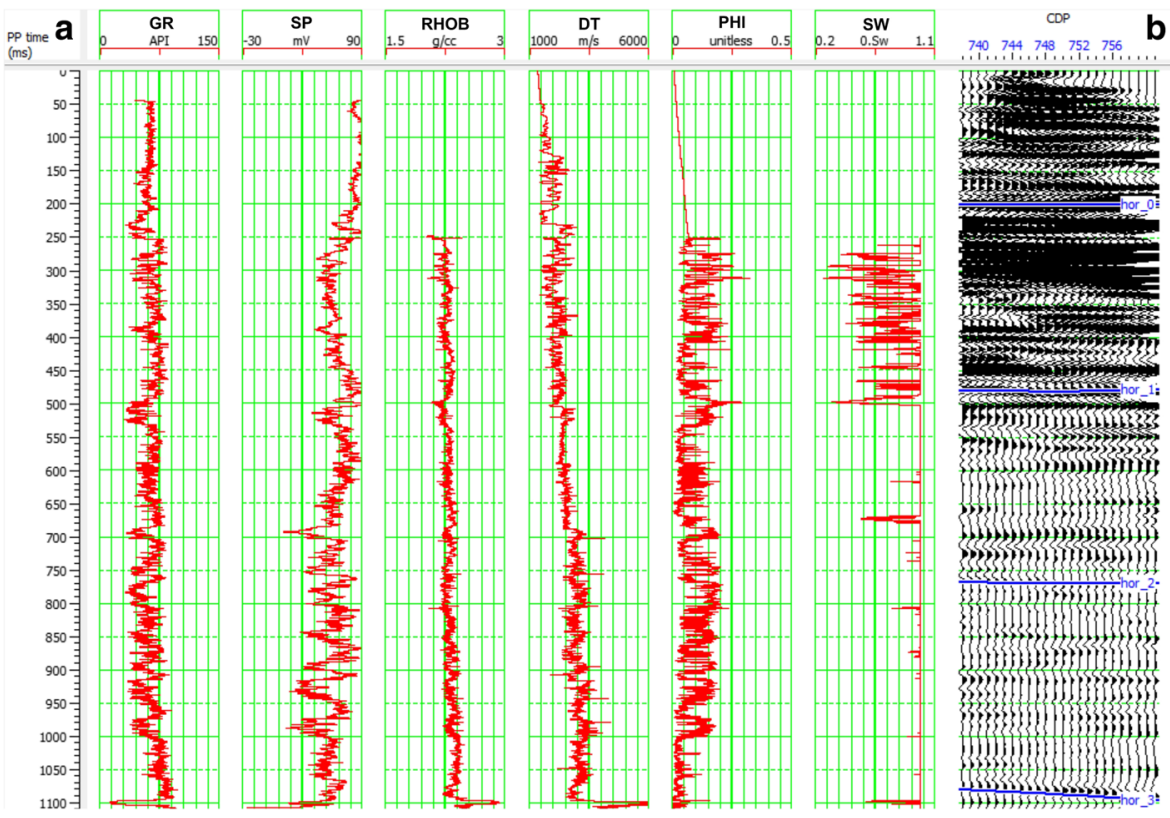

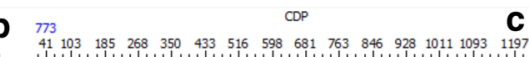

Fig. 13 Comparison of well $\log$ data and seismic data in the zone 1: a CHD-2 basic well logs (GR, SP, RHOB, DT, PHI, SW), b part of seismic section near the borehole and $\mathbf{c}$ the nearest $\mathrm{CDP}$ gather. SW decrease between 250 and $500 \mathrm{~ms}$ is the result of existing gas-bearing Miocene strata

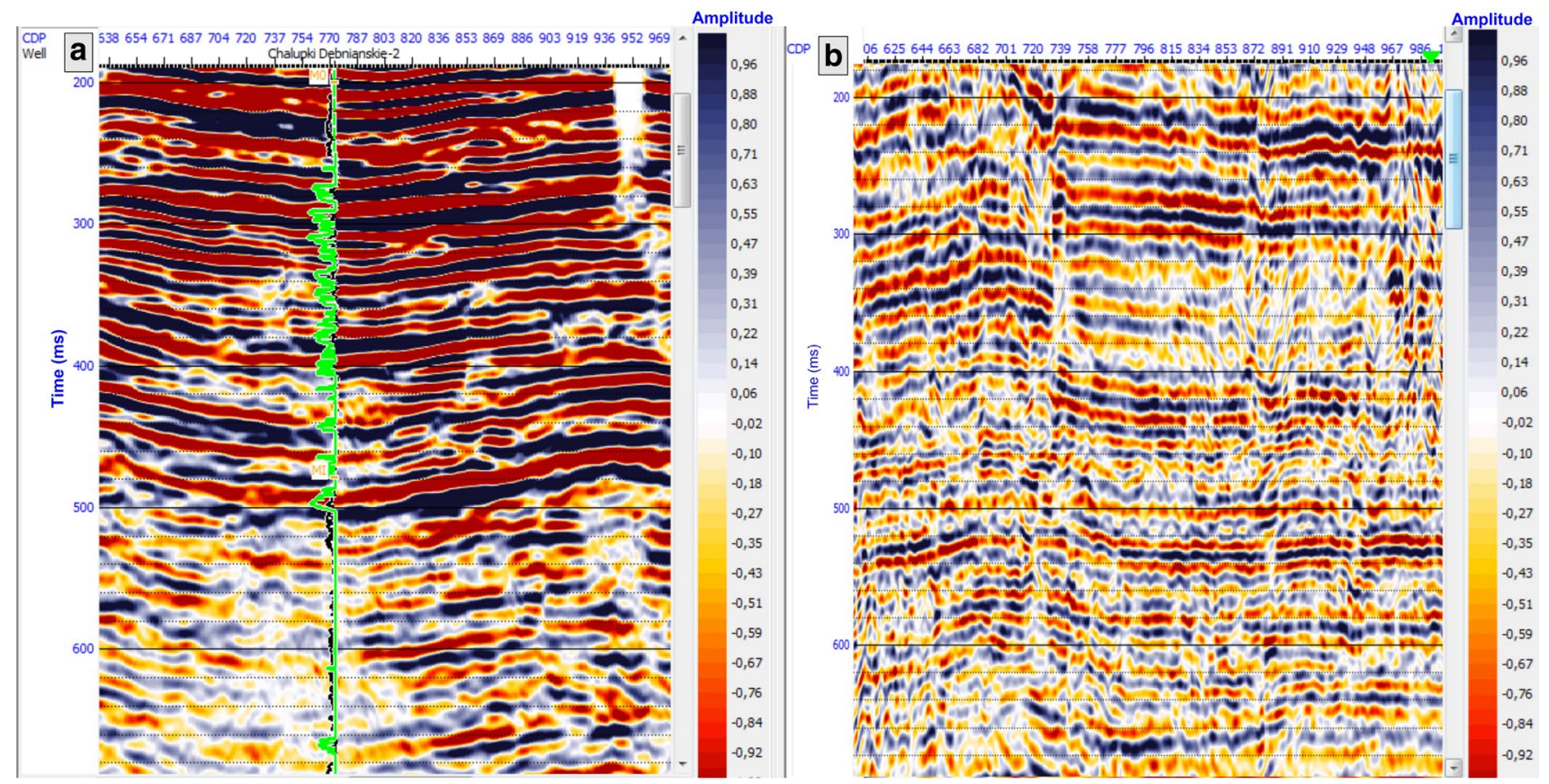

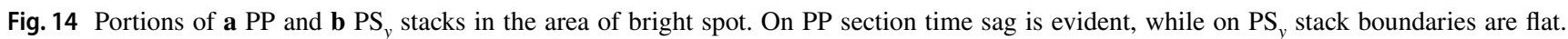
Additionally, on $\mathrm{PS}_{y}$ section boundaries under the reservoir are better mapped. PS sections are displayed in PP-wave travel time 
a

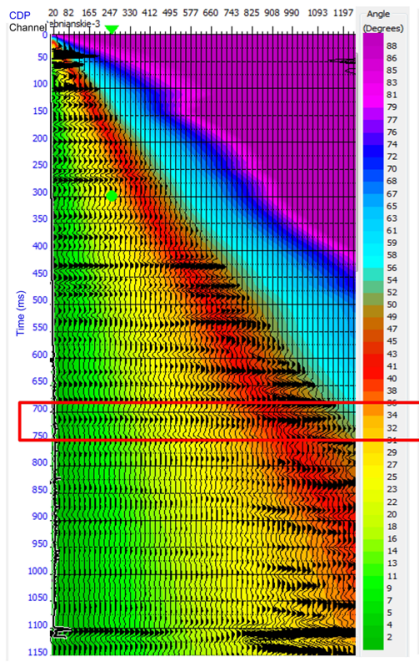

b

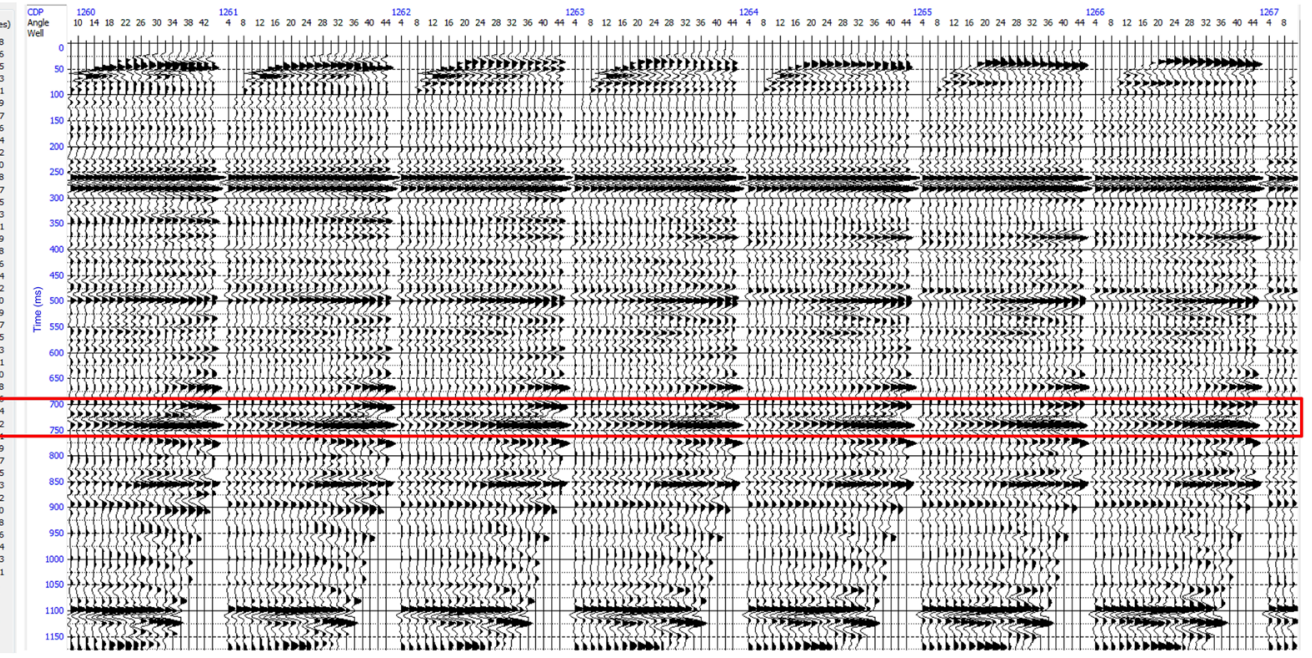

Fig. 15 a CRP gather in the neighborhood of CHD-3 well with color scale indicating incidence angle and $\mathbf{b}$ angle gathers. The interest area is inside the red rectangle

a

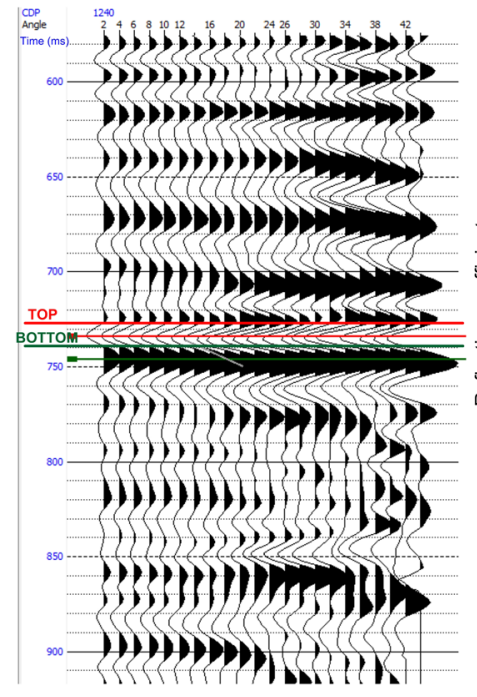

b

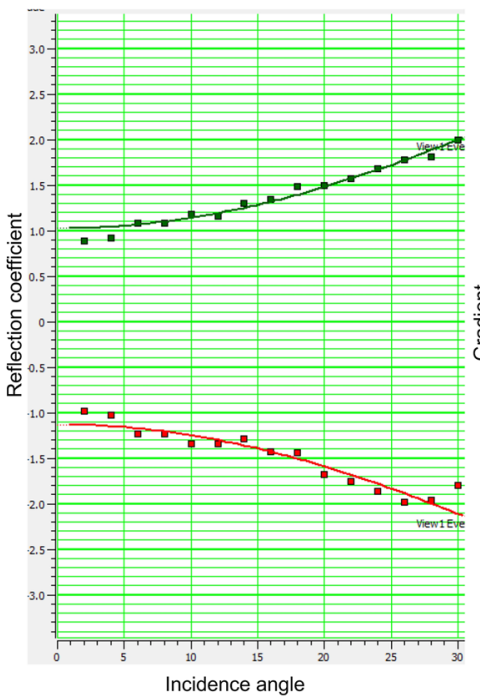

C

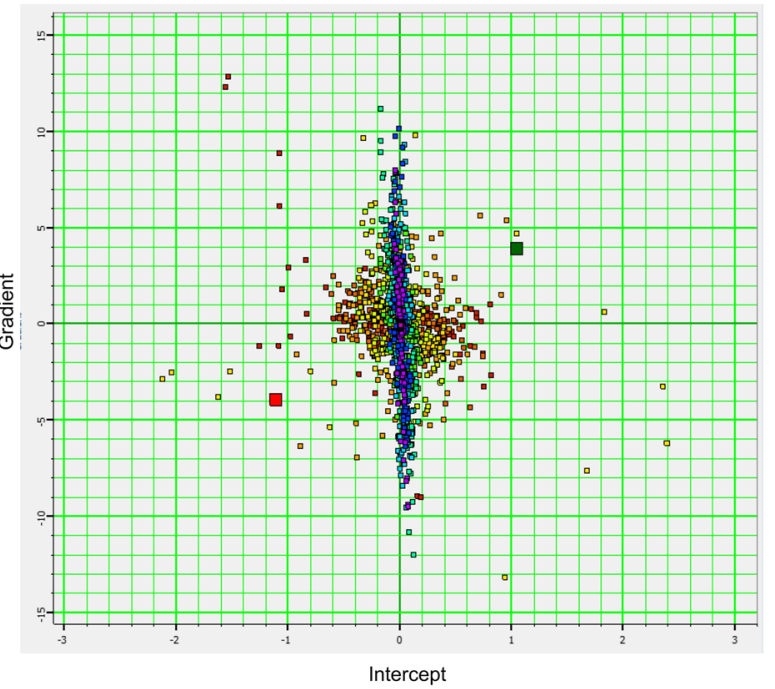

Fig. 16 AVA analysis for top (red) and bottom (green) of gas reservoir: a angle gather, $\mathbf{b}$ amplitude versus offset plot and $\mathbf{c}$ intercept/gradient crossplot. A typical third AVO class is visible. The two-term Aki-Richards approximation was used for calculations. The angle range was $1-30^{\circ}$

The development of the appropriate sequence of 3C seismic processing led to the results in the form of stacks and prestack gathers, which were appropriate for reservoir analyses based on the amplitude changes resulting from hydrocarbon saturation. On the basis of conducted analyses, we concluded that there is a strong amplitude anomaly in the central part of the conducted survey near $\mathrm{CH}-2$ well. All performed analyses proved the existence of many gasbearing layers. In the vicinity of $\mathrm{CH}-3$ well seismic anomaly around 1100-1300 CDP does not correlate with gas content information, in contrast to the AVA analysis results, which suggest clear and strong amplitude anomaly. Finally, in the third considered zone, chosen on the basis of PP RAP section, we did not observe any AVO anomaly suggesting oil or gas saturation. PS sections were helpful in verification of potential gas accumulations.

To summarize, obtained datasets with preserved relative amplitudes can be the basis for detailed seismic interpretation which can answer very important question: which of the perspective zones are worth drilling? On the datasets 


\section{Zone 1 Zone 2 Zone 3}

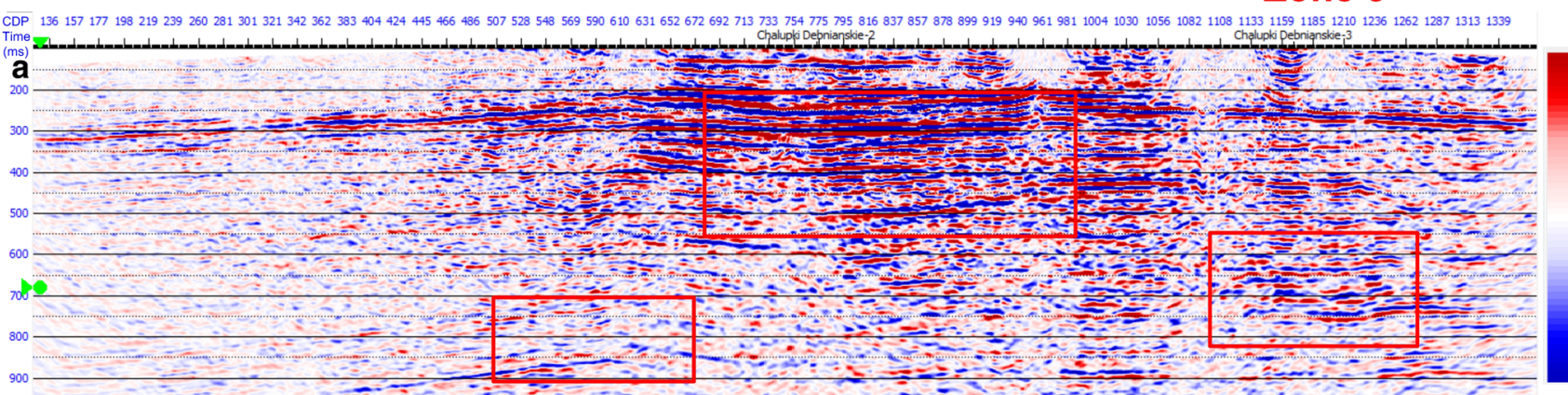

b
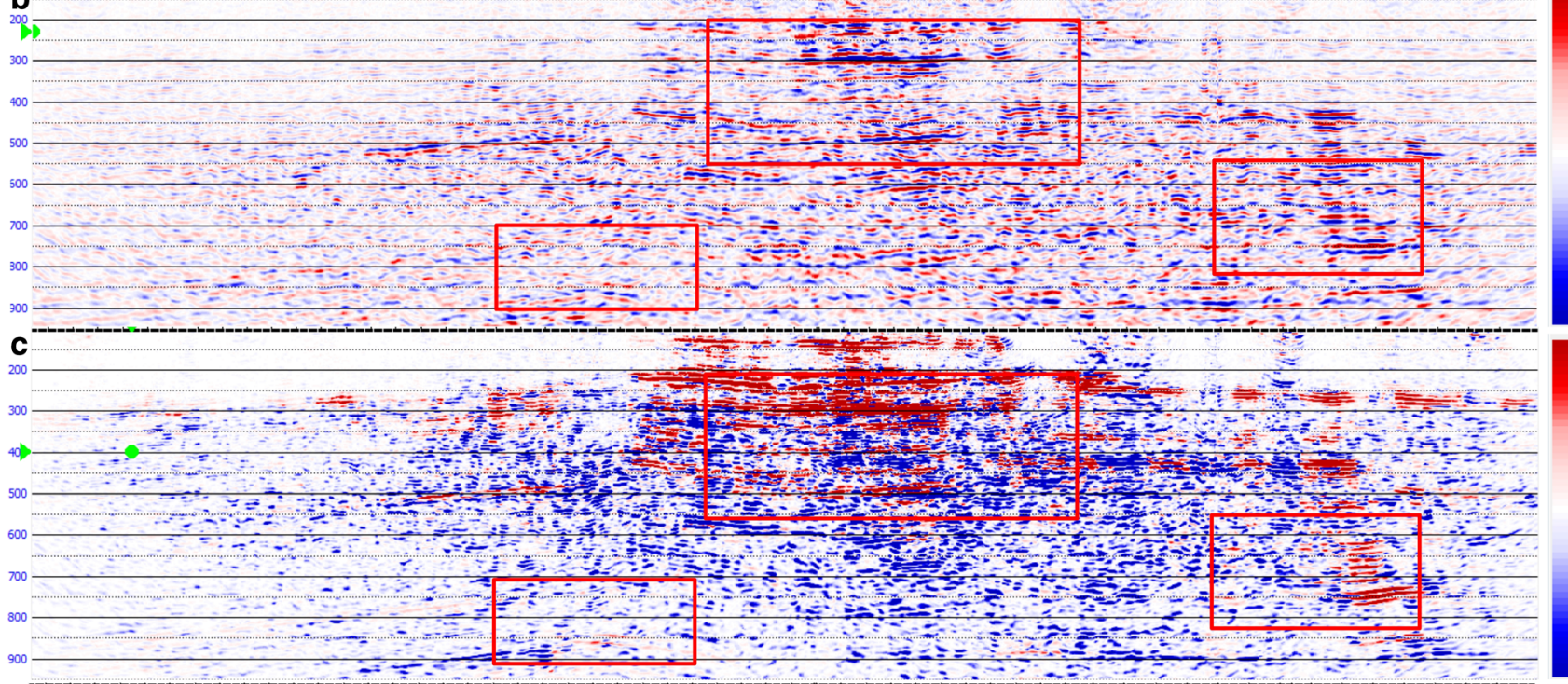

d

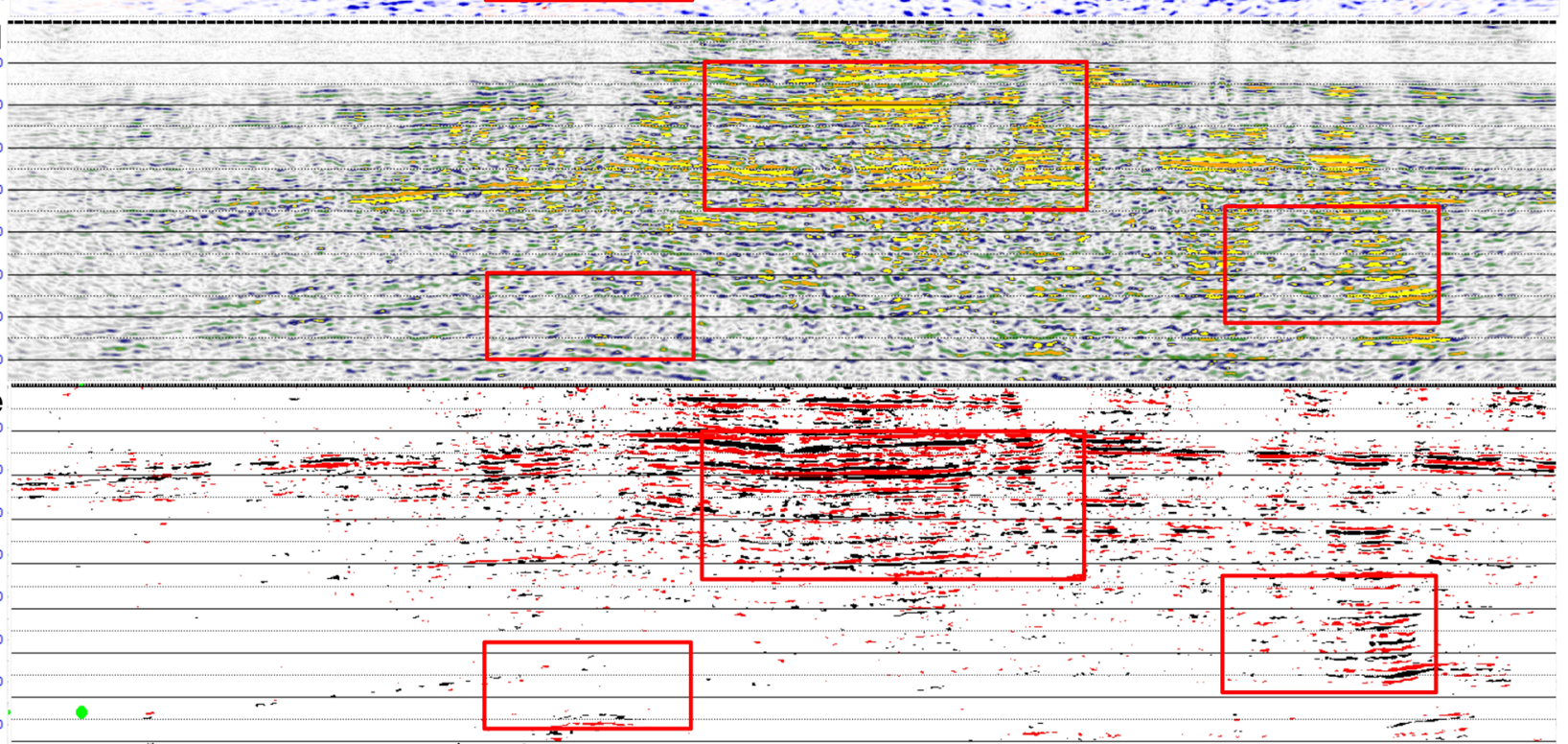

Fig. 17 Distribution of AVO attributes: a Intercept, b Gradient, $\mathbf{c}$ Product, $\mathbf{d}$ Scaled Poisson Ratio Change and e seismic section in RAP version. Reflection from the top of reservoir is red, reflection from the bottom-black (third AVO class) 
several reservoir analyses, including seismic attributes and AVO/AVA analysis, were conducted. The integrated analysis confirmed existence of reservoir which was characterized by good reservoir parameters (Chałupki Dębniańskie reservoir), as well as existence of non-productive zones which have been misinterpreted in previous studies.

Acknowledgements The paper was financially supported from the research subsidy no. 16.16.140.315 at the Faculty of Geology Geophysics and Environmental Protection of the AGH University of Science and Technology, Krakow, Poland, 2019. The paper was presented at the CAGG 2019 Conference "Challenges in Applied Geology and Geophysics" organized at the AGH University of Science and Technology, Krakow, Poland, 10-13 September 2019. The seismic processing presented in the paper was carried out as a part the master's thesis of Paweł Dubiel, Faculty of Geology, Geophysics and Environmental Protection, AGH, Kraków, Poland. Authors thank Polish Oil and Gas Company for providing seismic and well log data. Analyses were done in SeisSpace software (Landmark Halliburton) and Hampson-Russell software (CGG) thanks to Academic Grants funded by these companies to the AGH University of Science and Technology, Kraków, Poland.

\section{Compliance with ethical standards}

Conflict of interest On behalf of all authors, the corresponding author states that there is no conflict of interest.

Open Access This article is distributed under the terms of the Creative Commons Attribution 4.0 International License (http://creativeco mmons.org/licenses/by/4.0/), which permits unrestricted use, distribution, and reproduction in any medium, provided you give appropriate credit to the original author(s) and the source, provide a link to the Creative Commons license, and indicate if changes were made.

\section{References}

Aki K, Richards PG (1980) Quantitative seismology. W.H Freeman and Company, San Fransisco

Chopra S, Castagna JP (2014) AVO. Investigations in geophysics. Society of Exploration Geophysicists, Tulsa
Dubiel P (2018) Reprocessing of seismic line Chalupki Debnianskie 2D-3C for reservoir analysis. Master thesis, AGH-UST (in Polish with English abstract)

Dufour J (1996) Refraction static analysis of P-S seismic data using the plus-minus time analysis method. Master thesis, The University of Calgary

Farfour M, Yoon WJ (2016) A review on multicomponent seismology: a potential seismic application for reservoir characterization. $J$ Adv Res 7:515-524

Gaiser J (2016) 3C seismic and VSP: converted waves and vector wavefield applications. Society of Exploration Geophysicists, Tulsa

Gruszczyk E, Misiaczek P, Trześniowski Z (2002) Efficiency analysis of implementation of 2D-3C method by detection of the natural gas deposits in Miocene basin. Nafta-Gaz 58(9):477-485 (in Polish with English abstract)

Jezierska E, Keller-Utracka E (2003) Preparation of the results of Chałupki Dębniańskie 2D-3C seismic research. Geofizyka Kraków Sp. z o.o, Krakow (in Polish)

Mancini F, Li XY, Ziolkowski A, Pointer T (2002) Interpreting velocity ratios from $4 \mathrm{C}$ seismic data and well logs in the presence of gas and Anisotropy. SEG international exposition and 72nd annual meeting, Salt Lake City, Utah

Marzec P, Pietsch K, Kobylarski M (2006) Application of P and PS seismic sections in a calibration of S-wave velocity model. Geologia 32(4):419-440 (in Polish with English abstract)

Myśliwiec M (2004a) Exploration for gas accumulations in the Miocene deposits of the Carpathian Foredeep using Direct Hydrocarbon Indicators (southern Poland). Prz Geol 52:299-306 (in Polish with English abstract)

Myśliwiec M (2004b) The Miocene reservoir rocks of the Carpathian Foredeep. Prz Geol 52:581-592 (in Polish with English abstract)

Myśliwiec M (2004c) Traps for gas accumulations and the resulting zonation of the gas fields in the Miocene strata of the eastern part of the Carpathian Foredeep (SE Poland). Prz Geol 52:657-664 (in Polish with English abstract)

Stewart RR, Gaiser JE, Brown JR, Lawton DC (1999) Converted-wave seismic exploration: a tutorial. CREWES Research Report. https:// www.researchgate.net/publication/265347541_Converted-wave_ seismic_exploration_a_tutorial. Accessed 30 Apr 2019

Treitel S (1974) The complex Weiner filter. Geophysics 39(2):169-173

Yilmaz O (2001) Seismic data analysis. Society of Exploration Geophysicists, Tulsa 